\section{Uso de medicamentos antidepressivos na amamentação: avaliação da conformidade das bulas com fontes bibliográficas baseadas em evidências científicas}

\section{Use of antidepressants during breastfeeding: evaluating drug package inserts conformity with science-based bibliographical sources}

\section{Uso de medicamentos antidepresivos durante la lactancia: evaluación de la conformidad de los prospectos, respecto a fuentes bibliográficas basadas en evidencias científicas}

\section{Resumo}

O objetivo deste artigo foi avaliar a conformidade entre as recomendações de uso de medicamentos antidepressivos durante a amamentação, presentes em bulas, e as recomendações de fontes bibliográficas baseadas em evidências científicas. Foram avaliadas as bulas padrão de 23 antidepressivos com registro ativo no Brasil. A presença de contraindicação do uso do antidepressivo durante a amamentação foi comparada com as informações presentes no manual técnico do Ministério da Saúde, no livro Medications and Mothers' Milk e nas bases de dados LactMed, Micromedex e UpToDate. Na maioria das bulas (62,5\%), o antidepressivo é contraindicado na amamentação. Entre as fontes bibliográficas, esse percentual variou de 0\% a 25\%. O estudo aponta para baixa conformidade entre bulas e fontes bibliográficas, alertando sobre a necessidade de revisão do conteúdo e forma de apresentação das informações presentes nas bulas dos antidepressivos no Brasil.

Uso de Medicamentos; Antidepressivos; Bulas de Medicamentos;

Aleitamento Materno
COMUNICAÇÃO BREVE

BRIEF COMMUNICATION

\author{
Tatiane da Silva Dal Pizzol \\ Cassia Garcia Moraes 2 \\ Marceli Vilaverde Diello 1 \\ Paola Melo Campos 3 \\ Julia Tauana Pletsch 1 \\ Camila Giugliani 4
}

doi: $10.1590 / 0102-311 \times 00041018$

\author{
Correspondência \\ T. S. Dal Pizzol \\ Faculdade de Farmácia, Universidade Federal do Rio Grande \\ do Sul. \\ Av. Ipiranga 2752, 2 o andar (CIM), Porto Alegre, \\ RS 90610-000, Brasil. \\ tatiane.silva@ufrgs.br \\ 1 Faculdade de Farmácia, Universidade Federal do Rio Grande \\ do Sul, Porto Alegre, Brasil. \\ 2 Hospital Moinhos de Vento, Porto Alegre, Brasil. \\ 3 Escola de Enfermagem, Universidade Federal do Rio Grande \\ do Sul, Porto Alegre, Brasil. \\ 4 Faculdade de Medicina, Universidade Federal do Rio Grande \\ do Sul, Porto Alegre, Brasil.
}




\section{Introdução}

Durante o período em que a mulher amamenta é comum o uso de medicamentos 1,2. Estudos internacionais mostram que antidepressivos inibidores seletivos da recaptação da serotonina estão entre os dez fármacos mais utilizados na amamentação ${ }^{3}$, e que as desordens depressivas estão entre os principais motivos que levam ao uso de medicamentos neste período ${ }^{4}$. A decisão pelo uso seguro deve considerar diversos fatores: farmacocinéticos (i.e. via de administração, dose administrada, intervalo entre doses); características físico-químicas do fármaco (i.e. solubilidade e ligação a proteínas); e aspectos da amamentação (i.e. idade da criança, frequência das mamadas, tempo entre a tomada do medicamento e a mamada) 5 .

Para a busca de informações, tanto o profissional da saúde quanto a nutriz, acabam consultando, às vezes unicamente, as bulas dos medicamentos. Em vários países, as bulas são reconhecidas como uma importante ferramenta para a educação em saúde. Agências reguladoras de medicamentos como a Agência Nacional de Vigilância Sanitária (Anvisa) têm estabelecido padrões para a elaboração de bulas diferenciadas para o profissional da saúde e para o paciente.

O objetivo do estudo foi avaliar a conformidade das recomendações de uso durante a amamentação presentes em bulas de medicamentos antidepressivos com aquelas provenientes de fontes bibliográficas baseadas em evidências científicas.

\section{Método}

Foram selecionados os fármacos classificados como antidepressivos pela Anatomical Therapeutic Chemical (ATC), sistema desenvolvido pela Organização Mundial da Saúde (OMS) que classifica os medicamentos em grupos e subgrupos (http://www.whocc.no). Baseando-se nessa seleção, foram: (1) verificados quais fármacos eram registrados no Brasil, de acordo com informações disponíveis na página de Internet da Anvisa (http://www.anvisa.gov.br); (2) selecionados os medicamentos de referência para cada substância ativa; e (3) extraídas as bulas padrão do profissional no Bulário Eletrônico (http://www.anvisa.gov.br/datavisa/fila_bula/index.asp) da Anvisa, em agosto de 2017.

As informações sobre o uso na amamentação foram extraídas das seções Contraindicações ou Advertências e Precauções, de acordo com resolução da Anvisa 6.

As fontes de informação bibliográficas (Quadro 1) - manual técnico do Ministério da Saúde 7, livro Medications and Mothers' Milk 8 e as bases de dados LactMed (https://toxnet.nlm.nih.gov/newtoxnet/ lactmed.htm), Micromedex (https://www-micromedexsolutions-com.ez68.periodicos.capes.gov.br/ micromedex2/librarian) e UpToDate (https://www.uptodate.com/home) - foram selecionadas com base em estudo anterior 9 e consulta a especialistas. A classificação de risco em cada uma das fontes é apresentada no Quadro 2.

O desfecho foi a presença de informação contraindicando o uso do antidepressivo durante a amamentação. A informação foi classificada em "sim", quando a bula claramente contraindicava o uso durante a amamentação (ou contraindicava a amamentação durante o uso do medicamento), e "não", nas outras situações (uso compatível ou avaliação de risco/benefício). Para as fontes com sistema de classificação (o manual técnico do Ministério da Saúde, o livro e a base Micromedex), a contraindicação foi baseada nas categorias sinalizadas no Quadro 2. Nas outras fontes (as bases Lactmed e UpToDate), pela ausência de sistema de classificação, foi identificada no texto a recomendação explícita sobre a contraindicação. No LactMed, quando o texto recomendava a utilização de medicamento alternativo, sem mencionar claramente a possibilidade de uso concomitante, classificamos como contraindicado. No UpToDate, quando era mencionado que a informação provinha do laboratório fabricante, esta foi considerada na classificação.

Nas bulas em que foi identificada a contraindicação, foi avaliada a recomendação em relação à decisão entre não amamentar ou não utilizar o medicamento.

A classificação nas bulas foi realizada por dois revisores, de forma independente, e as discordâncias foram resolvidas por consenso. Para cada fonte foi verificada a concordância entre as classificações dos dois revisores. Calculou-se o total de medicamentos que receberam a mesma classificação pelos dois revisores, dividido pelo total de medicamentos avaliados naquela fonte. 


\section{Quadro 1}

Descrição das fontes de informação bibliográficas utilizadas e relação dos 23 medicamentos incluídos no estudo.

\begin{tabular}{|c|c|}
\hline \multicolumn{2}{|r|}{ FONTES BIBLIOGRÁFICAS UTILIZADAS } \\
\hline $\begin{array}{l}\text { Manual técnico do Ministério da } \\
\text { Saúde } 7\end{array}$ & $\begin{array}{l}\text { Composto da revisão sobre fármacos e outras substâncias transferidas para o leite materno e seus } \\
\text { possíveis efeitos no lactente e/ou na lactação, tendo como referência as publicações da Academia } \\
\text { Americana de Pediatria (AAP), da Organização Mundial da Saúde (OMS) e do livro Medications and Mothers' } \\
\text { Milk (edição de 2008). Conta com sistema de classificação em três níveis. } \\
\text { Local de busca: seção Fármacos que Atuam no Sistema Nervoso Central. }\end{array}$ \\
\hline Medications and Mother's Milk 8 & $\begin{array}{l}\text { Livro do autor T. W. Hale, atualizado a cada dois anos, dispõe de monografias sobre uma ampla variedade } \\
\text { de medicamentos e substâncias naturais. Características farmacológicas relevantes, pesquisas primárias, } \\
\text { bem como a classificação da AAP estão incluídas nas monografias. Utiliza sistema de classificação numérica } \\
\text { de cinco pontos para cada medicamento. } \\
\text { Local de busca: monografia do medicamento. }\end{array}$ \\
\hline $\begin{array}{l}\text { LactMed (https://toxnet.nlm.nih. } \\
\text { gov/newtoxnet/lactmed.htm) }\end{array}$ & $\begin{array}{l}\text { Recurso online, elaborado por painel de especialistas com base na literatura científica e atualizado } \\
\text { mensalmente. Inclui informações sobre os níveis do fármaco no leite materno, efeitos na criança e efeitos } \\
\text { sobre a lactação e leite materno. Não apresenta sistema de classificação, mas sim um resumo descritivo do } \\
\text { uso do medicamento durante a lactação e amamentação. } \\
\text { Local de busca: monografia do medicamento > Drug Levels and Effects > Summary of Use during Lactation. }\end{array}$ \\
\hline $\begin{array}{l}\text { Micromedex (https://www- } \\
\text { micromedexsolutions-com. } \\
\text { ez68.periodicos.capes.gov.br/ } \\
\text { micromedex2/librarian) }\end{array}$ & $\begin{array}{l}\text { Recurso online comumente usado em serviços de informação sobre medicamentos, constituído de } \\
\text { monografias contendo indicações aprovadas pela Agência Americana de Alimentos e Medicamentos (FDA), } \\
\text { usos off-label e informações sobre a segurança do medicamento, entre outras informações. Apresenta } \\
\text { classificação própria sobre a segurança do uso dos medicamentos na amamentação, dividida em } \\
\text { quatro categorias, feita com base nas evidências disponíveis e/ou consenso de especialistas. Apresenta } \\
\text { classificação da AAP. } \\
\text { Local de busca: monografia do medicamento > Medication Safety > Pregnancy \& Lactation. }\end{array}$ \\
\hline $\begin{array}{l}\text { UpToDate (https://www. } \\
\text { uptodate.com/home) }\end{array}$ & $\begin{array}{l}\text { Recurso online muito utilizado na prática clínica pelos profissionais médicos nos ambientes hospitalar } \\
\text { e ambulatorial. É considerada uma das fontes mais atualizadas, que faz um apanhado das evidências } \\
\text { disponíveis para apoiar a tomada de decisão do profissional, que levará em conta também a singularidade } \\
\text { de cada situação clínica. Não apresenta sistema de classificação, mas sim um resumo descritivo do uso do } \\
\text { medicamento durante a lactação e a amamentação e recomendações de condutas. } \\
\text { Local de busca: monografia do medicamento. }\end{array}$ \\
\hline \multicolumn{2}{|r|}{ MEDICAMENTOS INCLUÍDOS NA PESQUISA * } \\
\hline $\begin{array}{l}\text { Inibidores da recaptação de } \\
\text { monoamina não seletivos } \\
\text { (N06AA) }\end{array}$ & $\begin{array}{l}\text { Amitriptilina (N06AA09); Clomipramina (N06AA04); Imipramina (N06AA02); Maprotilina (N06AA21); } \\
\text { Nortriptilina (N06AA10) }\end{array}$ \\
\hline $\begin{array}{l}\text { Inibidores seletivos da } \\
\text { recaptação de serotonina } \\
\text { (N06AB) }\end{array}$ & $\begin{array}{c}\text { Citalopram (N06AB04); Escitalopram (N06AB10); Fluoxetina (N06AB03); Fluvoxamina (N06AB08); Paroxetina } \\
\text { (N06AB05); Sertralina (N06AB06) }\end{array}$ \\
\hline $\begin{array}{l}\text { Inibidores da monoamina } \\
\text { oxidase, não seletivo (N06AF) }\end{array}$ & Tranilcipromina (N06AF04) \\
\hline $\begin{array}{l}\text { Inibidores da monoamina } \\
\text { oxidase (N06AG) }\end{array}$ & Moclobemida (N06AG02) \\
\hline Outros (N06AX) & $\begin{array}{l}\text { Agomelatina (N06AX22); Bupropiona (N06AX12); Desvenlafaxina (N06AX23); Duloxetina (N06AX21); } \\
\text { Mirtazapina (N06AX11); Reboxetina (N06AX18); Tianeptina (N06AX14); Trazodona (N06AX05); Venlafaxina } \\
\text { (N06AX16); Vortioxetina (N06AX26) }\end{array}$ \\
\hline
\end{tabular}

* De acordo com o sistema de classificação ATC (Anatomical Therapeutic Chemical; http://www.whocc.no), o primeiro nível da classificação (letra N) corresponde ao principal grupo anatômico ( $\mathrm{N}$ = sistema nervoso), o segundo nível (dois algarismos) corresponde ao subgrupo terapêutico (06 = psicoanalépticos), o terceiro (letra) corresponde ao subgrupo farmacológico ( $\mathrm{A}$ = antidepressivos), o quarto (letra) corresponde ao subgrupo químico ( $A$ = Inibidores da recaptação de monoamina não seletivos; $B$ = Inibidores seletivos da recaptação de serotonina; $F=$ Inibidores da monoamina oxidase, não seletivo; $G$ = Inibidores da monoamina oxidase; $X$ = outros antidepressivos), o quinto nível (dois algarismos) corresponde à substância ativa (por exemplo, N06AA02 = imipramina). 


\section{Quadro 2}

Classificação da compatibilidade de uso dos medicamentos antidepressivos durante a amamentação em cada uma das fontes de informação pesquisadas *

\begin{tabular}{|c|c|}
\hline FONTE DE INFORMAÇÃO & CLASSIFICAÇÃO DE RISCO ** \\
\hline \multirow{3}{*}{$\begin{array}{l}\text { Manual técnico do } \\
\text { Ministério da Saúde } 7 \\
\text { (Brasil) }\end{array}$} & $\begin{array}{l}\text { 1. Compatível com a amamentação: fármacos cujo uso é potencialmente seguro durante a lactação, haja vista } \\
\text { não haver relatos de efeitos farmacológicos significativos para o lactente. }\end{array}$ \\
\hline & $\begin{array}{l}\text { 2. Uso criterioso durante a amamentação: medicamentos cujo uso no período da lactação depende da } \\
\text { avaliação do risco/benefício. Quando utilizados, exigem monitorização clínica e/ou laboratorial do lactente, } \\
\text { devendo ser usados durante o menor tempo e na menor dose possível. Novos medicamentos, cuja segurança } \\
\text { durante a amamentação ainda não foi devidamente documentada, encontram-se nesta categoria. }\end{array}$ \\
\hline & $\begin{array}{l}\text { 3. Uso contraindicado durante a amamentação: medicamentos que exigem a interrupção da amamentação } \\
\text { pelas evidências ou risco significativo de efeitos colaterais importantes no lactente. }\end{array}$ \\
\hline \multirow[t]{5}{*}{$\begin{array}{l}\text { Medications and Mothers' } \\
\text { Milk } 8 \text { (Estados Unidos) }\end{array}$} & $\begin{array}{l}\text { L1 Compatível: medicamento que tem sido utilizado por um grande número de mães que amamentam, } \\
\text { sem qualquer aumento observado em efeitos adversos na criança. Estudos controlados em mulheres que } \\
\text { amamentam não demonstram um risco para a criança e a possibilidade de danos para a criança; ou o produto } \\
\text { não é biodisponível para uma criança. }\end{array}$ \\
\hline & $\begin{array}{l}\text { L2 Provavelmente Compatível: medicamento que tem sido estudado em um número limitado de mulheres } \\
\text { que amamentam sem um aumento dos efeitos adversos na criança, e/ou a evidência de um risco demonstrado } \\
\text { com o uso deste medicamento em uma mulher que amamenta é remoto. }\end{array}$ \\
\hline & $\begin{array}{l}\text { L3 Provavelmente Compatível: não há estudos controlados em mulheres que amamentam, no entanto, o } \\
\text { risco de efeitos adversos para a criança amamentada é possível, ou estudos controlados mostram que efeitos } \\
\text { adversos não são graves, apenas mínimos. Os medicamentos devem ser dados somente se o benefício potencial } \\
\text { justificar o risco potencial para a criança. (Novos medicamentos que não têm absolutamente nenhum dado } \\
\text { publicado são automaticamente classificados nesta categoria, independentemente de quão seguro eles podem } \\
\text { ser). }\end{array}$ \\
\hline & $\begin{array}{l}\text { L4 Possivelmente Perigoso: não há evidência de risco para o bebê ou para a produção de leite materno, mas os } \\
\text { benefícios provenientes do uso em mães que amamentam podem ser aceitáveis apesar do risco para a criança } \\
\text { (por exemplo, se o medicamento é necessário em uma situação de risco de vida ou de uma doença grave para } \\
\text { os quais as drogas mais seguras não podem ser utilizadas ou são ineficazes.) }\end{array}$ \\
\hline & $\begin{array}{l}\text { L5 Perigoso: o risco de usar o medicamento em mulheres amamentando claramente supera qualquer possível } \\
\text { benefício da amamentação. O medicamento é contraindicado em mulheres que estão amamentando um bebê. }\end{array}$ \\
\hline \multirow{4}{*}{$\begin{array}{l}\text { Micromedex (https://www- } \\
\text { micromedexsolutions-com. } \\
\text { ez68.periodicos.capes.gov. } \\
\text { br/micromedex2/librarian) } \\
\text { (Estados Unidos) }\end{array}$} & $\begin{array}{l}\text { 1. Risco para a criança não pode ser descartado: evidência disponível e/ou o consenso de peritos é } \\
\text { inconclusiva ou inadequada para determinar o risco para a criança quando o medicamento é usado durante a } \\
\text { amamentação. Pesar os benefícios potenciais do tratamento medicamentoso contra os riscos potenciais antes } \\
\text { de se prescrever o fármaco durante a amamentação. }\end{array}$ \\
\hline & $\begin{array}{l}\text { 2. Risco para a criança tem sido demonstrado: evidência e/ou consenso de peritos tem demonstrado } \\
\text { efeitos de dano infantil quando o medicamento é usado durante a amamentação. Uma alternativa para esse } \\
\text { fármaco deverá ser prescrita ou as pacientes deverão ser aconselhadas a descontinuar o fármaco durante a } \\
\text { amamentação. }\end{array}$ \\
\hline & $\begin{array}{l}\text { 3. Risco para a criança é mínimo: o nível de evidência e/ou o consenso de peritos sugere que este fármaco } \\
\text { oferece risco mínimo para o bebê quando usado durante a amamentação. }\end{array}$ \\
\hline & $\begin{array}{l}\text { 4. Efeitos sobre o leite são possíveis: evidência sugere que este fármaco possa alterar a produção ou } \\
\text { composição do leite. Se uma alternativa a esse fármaco não é prescrita, deve-se monitorar o bebê quanto a } \\
\text { efeitos adversos e/ou ingestão adequada de leite. }\end{array}$ \\
\hline
\end{tabular}

\footnotetext{
* As categorias sinalizadas em cinza foram consideradas aquelas que contraindicam o medicamento na amamentação;

** LactMed e UpToDate não contam com sistema de classificação.
} 


\section{Resultados}

Houve elevada concordância entre as classificações dos dois revisores (de 91 a 100\%, dependendo da fonte avaliada). A Tabela 1 apresenta a classificação dos medicamentos quanto à contraindicação do uso durante a amamentação nas bulas e nas cinco fontes. Entre as 23 bulas, 56,5\% claramente contraindicavam o uso concomitante. Quando avaliadas apenas as bulas constantes em todas as fontes $(\mathrm{n}=16)$, o percentual foi de $62,5 \%$, superior a qualquer um dos percentuais alcançados nas fontes (que variou de $0 \%$ a $25 \%$ ).

Das 13 bulas que contraindicavam o uso concomitante, 5 (38,5\%) recomendavam que mães sob tratamento não deveriam amamentar e 8 (61,5\%) recomendavam optar pela não utilização do medicamento ou pela amamentação.

\section{Tabela 1}

Classificação dos medicamentos quanto à contraindicação de uso durante a amamentação nas bulas dos profissionais e nas cinco fontes avaliadas * .

\begin{tabular}{|c|c|c|c|c|c|c|c|c|c|c|c|c|}
\hline \multirow[t]{2}{*}{$\begin{array}{l}\text { Contraindicação } \\
\text { do uso do } \\
\text { antidepressivo }\end{array}$} & \multicolumn{2}{|c|}{$\begin{array}{l}\text { Bula do } \\
\text { profissional } \\
(n=23)\end{array}$} & \multicolumn{2}{|c|}{$\begin{array}{l}\text { Manual técnico } \\
\text { do Ministério da } \\
\text { Saúde }(n=16)\end{array}$} & \multicolumn{2}{|c|}{$\begin{array}{c}\text { Medications and } \\
\text { Mothers' Milk } \\
(\mathrm{n}=18)\end{array}$} & \multicolumn{2}{|c|}{$\begin{array}{l}\text { LactMed } \\
(\mathrm{n}=21)\end{array}$} & \multicolumn{2}{|c|}{$\begin{array}{l}\text { Micromedex } \\
(n=21)\end{array}$} & \multicolumn{2}{|c|}{$\begin{array}{l}\text { UpToDate } \\
(n=20)\end{array}$} \\
\hline & n/total ** & $\%$ & n/total ** & $\%$ & n/total ** & $\%$ & n/total ** & $\%$ & n/total ** & $\%$ & n/total ** & $\%$ \\
\hline $\begin{array}{l}\text { Todos os } \\
\text { antidepressivos na } \\
\text { fonte }\end{array}$ & $13 / 23$ & 56,5 & $0 / 16$ & 0,0 & $0 / 18$ & 0,0 & $2 / 21$ & 9,5 & $1 / 21$ & 4,8 & $6 / 20$ & 30,0 \\
\hline $\begin{array}{l}\text { Inibidores da } \\
\text { recaptação de } \\
\text { monoamina não } \\
\text { seletivos *** }\end{array}$ & $4 / 5$ & 80,0 & $0 / 5$ & 0,0 & $0 / 5$ & 0,0 & $0 / 5$ & 0,0 & $0 / 5$ & 0,0 & $2 / 5$ & 40,0 \\
\hline $\begin{array}{l}\text { Inibidores seletivos } \\
\text { da receptação de } \\
\text { serotonina \# }\end{array}$ & $2 / 6$ & 33,3 & $0 / 6$ & 0,0 & $0 / 6$ & 0,0 & $0 / 6$ & 0,0 & $1 / 6$ & 16,7 & $1 / 6$ & 16,7 \\
\hline $\begin{array}{l}\text { Outros } \\
\text { antidepressivos \#\# }\end{array}$ & $7 / 12$ & 58,3 & $0 / 5$ & 0,0 & $0 / 7$ & 0,0 & $2 / 10$ & 20,0 & $0 / 10$ & 0,0 & $3 / 9$ & 33,3 \\
\hline $\begin{array}{l}\text { Antidepressivos } \\
\text { presentes em todas } \\
\text { as fontes consultadas } \\
(\mathrm{n}=16) \# \# \#\end{array}$ & $10 / 16$ & 62,5 & $0 / 16$ & 0,0 & $0 / 16$ & 0,0 & $0 / 16$ & 0,0 & $1 / 16$ & 6,2 & $4 / 16$ & 25,0 \\
\hline $\begin{array}{l}\text { Inibidores da } \\
\text { recaptação de } \\
\text { monoamina não } \\
\text { seletivos *** }\end{array}$ & $4 / 5$ & 80,0 & $0 / 5$ & 0,0 & $0 / 5$ & 0,0 & $0 / 5$ & 0,0 & $0 / 5$ & 0,0 & $2 / 5$ & 40,0 \\
\hline $\begin{array}{l}\text { Inibidores seletivos } \\
\text { da recaptação de } \\
\text { serotonina \# }\end{array}$ & $2 / 6$ & 33,3 & $0 / 6$ & 0,0 & $0 / 6$ & 0,0 & $0 / 6$ & 0,0 & $1 / 6$ & 16,7 & $1 / 6$ & 16,7 \\
\hline $\begin{array}{l}\text { Outros } \\
\text { antidepressivos \#\# }\end{array}$ & $4 / 5$ & 80,0 & $0 / 5$ & 0,0 & $0 / 5$ & 0,0 & $0 / 5$ & 0,0 & $0 / 5$ & 0,0 & $1 / 5$ & 20,0 \\
\hline
\end{tabular}

* Dos 23 medicamentos avaliados, 7 não constavam no manual técnico do Ministério da Saúde, 2 no Micromedex, 5 no Medications and Mothers' Milk, 2 no LactMed. O UpToDate não trazia informações sobre 1 dos medicamentos e sobre o uso na amamentação para os outros 2;

** $\mathrm{n}$ /total = número de antidepressivos classificados como contraindicados durante a amamentação dividido pelo total de antidepressivos avaliados em cada fonte de informação, conforme disponibilidade. Por exemplo, do total de antidepressivos avaliados (23), 21 constavam na LactMed, destes,

2 apresentavam contraindicação;

*** Imipramina, clomipramina, amitriptilina, nortriptilina, maprotilina;

\# Fluoxetina, citalopram, paroxetina, sertralina, fluvoxamina, escitalopram;

\#\# Tranilcipromina, moclobemida, trazodona, mirtazapina, bupropiona, tianeptina, venlafaxina, reboxetina, duloxetina, desvenlafaxina, agomelatina, vortioxetina;

\#\#\# Avaliados apenas os antidepressivos presentes em todas as fontes de informação, totalizando 16 medicamentos. 


\section{Discussão}

O estudo revela a existência de expressivas inconsistências entre as bulas e as fontes bibliográficas consultadas. Nenhuma bula apontou claramente para a compatibilidade do uso do medicamento na amamentação; na maioria, a recomendação é de não fazer uso concomitante, contraindicando o medicamento ou a amamentação. De acordo com as fontes, a maioria dos antidepressivos é compatível com a amamentação, não havendo contraindicação explícita para o uso concomitante. A verificação de informações inconsistentes entre as bulas e as evidências científicas para outras classes de medicamentos foi relatada previamente 10,11 .

Entre as explicações para esses resultados, podemos considerar: (1) a existência de controvérsias na literatura, decorrente dos sistemas de classificação e das evidências utilizadas; (2) a priorização de informação conservadora nas bulas, resguardando o fabricante contra possíveis ações legais em detrimento de informação baseada nas melhores evidências. Para Davanzo et al. 12, o fabricante costuma contraindicar o uso de medicamentos durante a amamentação quando as informações são limitadas, exercendo, possivelmente, uma influência negativa para profissionais e nutrizes em relação ao início ou à continuidade da amamentação. Nesse sentido, as precauções não são, necessariamente, fruto de evidências, mas sim de medida defensiva do fabricante quando as informações de segurança não estão disponíveis 13, ou estão disponíveis, mas são controversas. É possível que as agências reguladoras de medicamentos, por sua vez, não estejam contribuindo satisfatoriamente para a minimização das informações precárias sobre esse tópico, como destacado por Arguello et al. ${ }^{14}$. Embora as bulas sejam produzidas pelas empresas farmacêuticas, elas são, em última instância, aprovadas pelas agências reguladoras, tornando-se uma fonte de informação oficial sobre medicamentos 14 .

A expressiva diferença observada entre as bulas e as fontes traz preocupação se considerarmos que a bula pode ser utilizada como a primeira fonte de informação, senão a única, para a tomada de decisão sobre a prescrição ou não do antidepressivo. A escolha de uma fonte de informação adicional pode levar a decisões diferentes, dependendo da fonte selecionada. Enquanto fontes respaldadas pela literatura estimulam a amamentação, aquelas que mencionam recomendações do fabricante contribuem para uma decisão mais conservadora. Em situações de dúvidas, o manual técnico do Ministério da Saúde e o LactMed estimulam a amamentação, respaldados pela literatura. Por outro lado, fontes como o UpToDate, que mencionam as recomendações do fabricante, contribuem para uma decisão mais conservadora, à semelhança das bulas brasileiras.

A informação das bulas de antidepressivos pode incentivar a interrupção da amamentação ou a não utilização de medicamentos que poderiam beneficiar a saúde e o bem-estar da nutriz 10. Um estudo qualitativo identificou o medicamento como fator de risco para o desmame precoce 15 .

Estimativas apontam para o aumento da prevalência e duração da amamentação, principalmente a exclusiva 16. Nesse contexto, informações acuradas sobre a segurança do uso de medicamentos nesse período devem estar acessíveis, permitindo a tomada de decisões informadas, de forma a preservar a amamentação e promover saúde para mães e filhos.

Os resultados apontam para a necessidade de as indústrias farmacêuticas e a agência reguladora revisarem seus posicionamentos sobre conteúdo e forma de apresentação das informações presentes nas bulas dos antidepressivos. Recomenda-se que fontes bibliográficas que trazem evidências com base em estudos com nutrizes sejam amplamente divulgadas, tornem-se mais acessíveis aos profissionais e que sejam periodicamente atualizadas. Aos profissionais, sugere-se a adoção de estratégias de minimização de risco para compatibilizar a amamentação com o uso de medicamentos (i.e. utilização da dose mínima eficaz e ajuste dos horários de administração). 


\section{Colaboradores}

T. S. Dal Pizzol, C. G. Moraes e C. Giugliani participaram da concepção do estudo, análise e interpretação dos resultados, redação e revisão crítica do conteúdo intelectual e aprovação da versão final do manuscrito. M. V. Diello, P. M. Campos e J. T. Pletsch participaram da coleta e análise dos dados, redação e revisão crítica do conteúdo intelectual e aprovação da versão final do manuscrito.

\section{Referências}

1. Schirm E, Schwagermann MP, Tobi H, de Jong-van den Berg LTW. Drug use during breastfeeding: a survey from the Netherlands. Eur J Clin Nutr 2004; 58:386-90.

2. Chaves RG, Lamounier JA, César CC. Selfmedication in nursing mothers and its influence on the duration of breastfeeding. J Pediatr (Rio J.) 2009; 85:129-34.

3. Stultz EE, Stokes JL, Shaffer ML, Paul IM, Berlin CM. Extent of medication use in breastfeeding women. Breastfeed Med 2007; 2:145-51.

4. Jayawickrama HS, Amir LH, Pirotta MV. GPs' decision-making when prescribing medicines for breastfeeding women: content analysis of a survey. BMC Res Notes 2010; 3:82.

5. Rowe H, Baker T, Hale TW. Maternal medication, drug use, and breastfeeding. Child Adolesc Psychiatr Clin N Am 2015; 24:1-20.

6. Agência Nacional de Vigilância Sanitária. Resolução - RDC no 47, de 8 de setembro de 2009. Estabelece regras para elaboração, harmonização, atualização, publicação e disponibilização de bulas de medicamentos para pacientes e para profissionais de saúde. Diário Oficial da União 2009; 9 set.

7. Ministério da Saúde. Amamentação e uso de medicamentos e outras substâncias. http:// bvsms.saude.gov.br/bvs/publicacoes/ama mentacao_uso_medicamentos_outras_subs tancias.pdf (acessado em 13/Nov/2018).

8. Hale TW, Rowe HE. Medications and mother's milk. 16th Ed. Plano: Hale Publishing; 2014.

9. Akus M, Bartick M. Lactation safety recommendations and reliability compared in 10 medication resources. Ann Pharmacother 2007; 41:1352-60.

\section{Informações adicionais}

ORCID: Tatiane da Silva Dal Pizzol (0000-00027566-7745); Cassia Garcia Moraes (0000-00025270-1883); Marceli Vilaverde Diello (0000-00019195-6670); Paola Melo Campos (0000-0001-58883836); Julia Tauana Pletsch (0000-0001-7526-3305); Camila Giugliani (0000-0002-2652-5214).

10. Chaves RG, Lamounier JA, César CC, Corradi MAL, Mello RP, Gontijo CM, et al. Amamentação e uso de antiinflamatórios não esteróides pela nutriz: informações científicas versus conteúdo em bulas de medicamentos comercializados no Brasil. Rev Bras Saúde Matern Infant 2006; 6:269-76.

11. Colaceci S, Giusti A, Chapin EM, Notarangelo M, De Angelis A, Vellone E, et al. The difficulties in antihypertensive drug prescription during lactation: is the information consistent? Breastfeed Med 2015; 10:468-73.

12. Davanzo R, Copertino M, De Cunto A, Minen F, Amaddeo A. Antidepressant drugs and breastfeeding: a review of the literature. Breastfeed Med 2011; 6:89-98.

13. Fortinguerra F, Clavenna A, Bonati M. Psychotropic drug use during breastfeeding: a review of the evidence. Pediatrics 2009; 124:e547-56.

14. Arguello B, Salgado TM, Fernandez-Llimos F. Assessing the information in the summaries of product characteristics for the use of medicines in pregnancy and lactation. Br J Clin Pharmacol 2015; 79:537-44.

15. Araújo OD, Cunha AL, Lustosa LR, Nery IS, Mendonça RCM, Campelo SMA. Aleitamento materno: fatores que levam ao desmame precoce. Rev Bras Enferm 2008; 61:488-92.

16. Victora CG, Bahl R, Barros AJD, França GVA, Horton S, Krasevec J, et al. Breastfeeding in the 21st century: epidemiology, mechanisms, and lifelong effect. Lancet 2016; 387:475-90. 


\section{Abstract}

This article sought to evaluate the conformity between recommendations regarding antidepressant use during breastfeeding found in drug package inserts with recommendations from science-based bibliographic sources. We evaluated the standard drug package inserts of 23 antidepressants with active registration in Brazil. The presence of contraindications of antidepressant use during breastfeeding was compared with information present in the Brazilian Ministry of Health technical manual, the book Medications and Mothers' Milk and on the databases LactMed, Micromedex and UpToDate. In most drug package inserts (62.5\%), antidepressants are contraindicated during breastfeeding. Among bibliographical sources, that percentage varied between 0\% and 25\%. The study shows a low conformity between drug package inserts and bibliographical sources, alerting to the need for revising the content and presentation of information present in antidepressant drug package inserts in Brazil.

Drug Utilization; Antidepressive Agents;

Medicine Package Inserts; Breast Feeding

\section{Resumen}

El objetivo de este artículo fue evaluar la conformidad entre las recomendaciones de uso de medicamentos antidepresivos durante la lactancia, presentes en prospectos, y las recomendaciones de fuentes bibliográficas, basadas en evidencias científicas. Se evaluaron los prospectos estándar de 23 antidepresivos con registro activo en Brasil. La presencia de contraindicaciones en el consumo de antidepresivos durante la lactancia se comparó con la información presente en el manual técnico del Ministerio de la Salud, en el libro Medications and Mothers' Milk, $y$ en las bases de datos LactMed, Micromedex y UpToDate. En la mayoría de los prospectos (62,5\%), el antidepresivo está contraindicado durante la lactancia. Entre las fuentes bibliográficas el porcentaje varió de $0 \%$ a $25 \%$. El estudio señala la escasa conformidad entre prospectos y fuentes bibliográficas, alertando sobre la necesidad de revisión del contenido, así como de la forma de presentación de la información que aparece en los prospectos de los antidepresivos en Brasil.

Utilización de Medicamentos; Antidepresivos; Prospectos de Medicamentos; Lactancia Materna
Recebido em 06/Mar/2018

Versão final reapresentada em 14/Nov/2018 Aprovado em 04/Dez/2018 